\title{
Characterization of Catechins in Water by Photoemission Yield Spectroscopy in Air
}

\author{
Daisuke YAMASHITA ${ }^{\dagger}$ and Atsushi IsHIZAKI \\ Research and Development Department, Riken Keiki Co., Ltd., 2-3 Minamisakae-cho, Kasukabe, \\ Saitama 344-0057, Japan
}

\begin{abstract}
Photoemission yield spectroscopy in air (PYSA) was applied for the characterization of catechins in water in ambient conditions. According to the results of measurements on aqueous solutions of epigallocatechin gallate (EGCg) of various concentrations, the photoemission yield is almost proportional to the concentration of EGCg. Contrarily, the threshold energy of photoemission, $E_{\mathrm{PET}}$, is almost constant at $5.46 \pm 0.02 \mathrm{eV}$. Moreover, we measured aqueous solutions of epicatechin (EC), epigallocatechin (EGC), and epicatechin gallate (ECg). The values of $E_{\mathrm{PET}}$ of EC, EGC, ECg were estimated to be $5.72 \pm 0.02,5.68 \pm 0.01$, and $5.45 \pm 0.02 \mathrm{eV}$, respectively, and a dependence on the molecular structure was found. Furthermore, changes in the photoemission yield spectra of heated EGCg were well explained by molecular orbital calculations on the basis of an assumption of epimerization.
\end{abstract}

Keywords Photoemission yield measurements, catechin, electronic structure, ambient conditions

(Received November 18, 2015; Accepted January 26, 2016; Published May 10, 2016)

\section{Introduction}

An open counter ${ }^{1-4}$ is a unique detector that can detect and count a small number of low-energy photoelectrons, and can be operated in air under atmospheric pressure. Therefore, photoemission yield measurements can be performed in air by using photoemission yield spectroscopy in air (PYSA), which employs an open counter as a detector. The observation of successive changes in a solid surface has been successfully performed. ${ }^{5-7}$ Note that, in principle, there is no limitation to applying PYSA to any other type of material. PYSA can easily detect photoelectrons from liquids or liquid surfaces.

Electron spectroscopies such as X-ray photoelectron spectroscopy (XPS), ultraviolet photoelectron spectroscopy (UPS), and Auger electron spectroscopy (AES) are widely used for the characterization of solid surfaces. However, these techniques have been performed in an ultrahigh vacuum, which limits the samples that can be analyzed. Pioneering work on electron spectroscopy experiments for liquids was performed by H. Siegbahn and K. Siegbahn. ${ }^{8}$ Although several attempts have been made, these experiments were conducted under vacuum conditions using dynamic samples such as a flowing liquid beam, ${ }^{8}$ flowing liquid jet, ${ }^{9}$ liquid films on conically shaped trundles, ${ }^{10}$ or rotating disks. ${ }^{11,12}$ Contrarily, photoemission experiments under ambient conditions have been realized by PYSA. It has been reported that PYSA is applicable to the in situ characterization of Langmuir layers of photosensitive molecules at the air/water interface in ambient conditions. ${ }^{13}$ In this study, we applied PYSA to photoemission experiments on liquids, focusing on components in beverages, under the same

$\dagger$ To whom correspondence should be addressed.

E-mail: d-yamashita@ rikenkeiki.co.jp conditions as our living environment.

Catechins, which are the main components of green tea polyphenols, have been extensively studied because of their various biological effects. ${ }^{14-28}$ Orders of activity of the epi-type (cis-type) catechins have been reported for some effects, e.g., antibacterial activity against Clostridium botulinum,,${ }^{14}$ inhibitory effects on the oxidative modification of low-density lipoproteins, ${ }^{15,16}$ inactivation effects on human type-A influenza virus, ${ }^{17}$ and growth inhibition of human lung cancer cell line. ${ }^{18}$ According to these reports, the gallic acid esters of catechins such as epicatechin gallate $(\mathrm{ECg})$ and epigallocatechin gallate (EGCg) show higher activities than catechins without a galloyl moiety such as epicatechin (EC) and epigallocatechin (EGC). On the other hand, it is known that green tea also contains a small amount of trans-type catechins, i.e., catechin (C), gallocatechin $(\mathrm{GC})$, catechin gallate $(\mathrm{Cg})$ and gallocatechin gallate $(\mathrm{GCg})$. The degradation of catechins, e.g., the epimerization of $\mathrm{EGCg}$ to $\mathrm{GCg}$, has attracted considerable attention. ${ }^{29-31}$ We then attempt to apply PYSA, which is sensitive to changes in the electronic structure around the highest occupied molecular orbital (HOMO), to the characterization of catechins in water. In this paper, changes in photoemission yield spectra due to the concentration or structure of a catechin are elucidated, mainly by using EGCg. The applicability of PYSA for characterizing the electronic properties of catechins in water under ambient conditions is demonstrated.

\section{Experimental}

\section{Samples}

Commercially available high-purity powders of (-)-epicatechin (98\%), (-)-epigallocatechin (98\%), (-)-epicatechin gallate $(98 \%)$, and (-)-epigallocatechin gallate $(90 \%)$, all obtained from 
<smiles>Oc1cc(O)c2c(c1)O[C@H](c1ccc(O)c(O)c1)[C@H](O)C2</smiles><smiles>Oc1cc(O)c2c(c1)O[C@H](c1cc(O)c(O)c(O)c1)[C@H](O)C2</smiles><smiles>O=C(O[C@H]1Cc2c(O)cc(O)cc2O[C@H]1c1ccc(O)c(O)c1)c1cc(O)c(O)c(O)c1</smiles><smiles>CCC(C)(Cl)c1cc(O)cc2c1C[C@H](OC(=O)c1cc(O)c(O)c(O)c1)[C@@H](c1cc(O)c(O)c(O)c1)O2</smiles>

Fig. 1 Structures of epicatechin (EC), epigallocatechin (EGC), epicatechin gallate (ECg) and epigallocatechin gallate (EGCg).

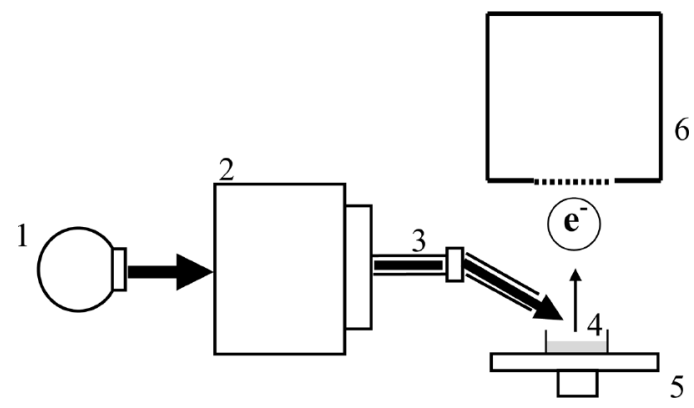

Fig. 2 Experimental setup for PYSA. 1, Deuterium lamp; 2, grating monochromator; 3 , optical fiber; 4 , sample; 5 , sample stage; 6 , open counter.

Wako Pure Chemicals, as shown in Fig. 1, were employed. In the photoemission yield experiment, catechins were mixed with pure water. After 5 min of agitation, the aqueous solution was dropped onto a container for the photoemission yield measurement. A stainless container dish $(10 \mathrm{~mm}$ diameter, $1 \mathrm{~mm}$ depth) was used. The aqueous solution was measured in air under atmospheric pressure.

\section{Apparatus}

Photoemission yield spectra were obtained using a photoemission yield spectrometer (AC-2, Riken Keiki). The experimental setup is shown in Fig. 2. UV light emitted from a deuterium lamp was monochromatized by a grating monochromator and focused on the surface of a sample through an optical fiber. Here, the energy of monochromatized UV light was swept at intervals of $0.1 \mathrm{eV}$ from 4.20 to $6.20 \mathrm{eV}$. The typical spectrum acquisition time was approximately $5 \mathrm{~min}$. The number of photoelectrons emitted from the surface of the sample was counted by a double cylindrical open counter, ${ }^{4}$ which was modified from the original open counter. ${ }^{1}$ The number of counter pulses per second produced at the anode is a function of the number of electrons emitted from the sample. A loss of counts is, however, introduced during the dead time of the counter. Then the number of electrons entered into the counter per second $N_{\text {in }}$ is expressed by the following equation: ${ }^{4}$

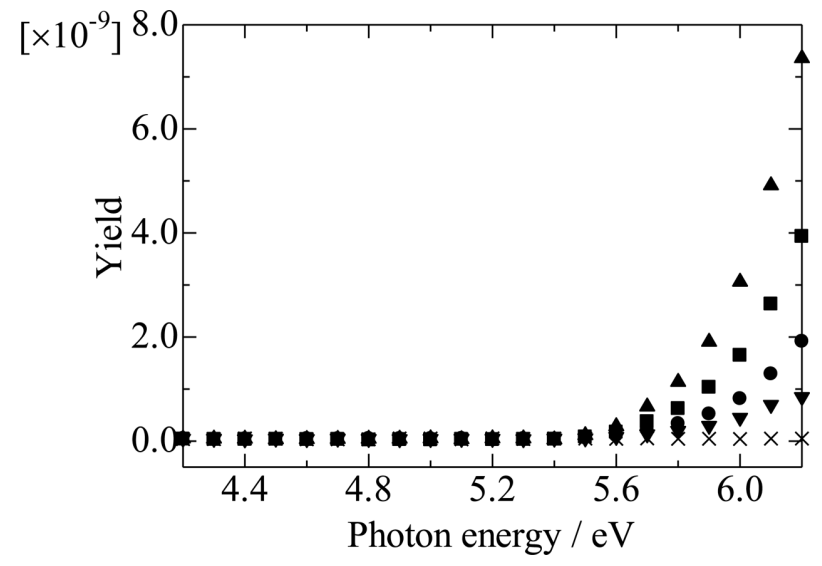

Fig. 3 Photoemission yield spectra of epigallocatechin gallate at the concentration of $1000(\boldsymbol{\Delta}), 500(\boldsymbol{\nabla}), 250(\boldsymbol{\bullet})$, and $100(\boldsymbol{\nabla}) \mathrm{mg} / \mathrm{L}$. The " $\times$ " symbol denotes the photoemission spectrum of the pure water surface.

$$
N_{\text {in }}=N_{\text {em }} f=\frac{N_{\text {obs }}}{1-\tau N_{\text {obs }}} .
$$

Here, $N_{\mathrm{em}}$ is the number of electrons emitted from the sample per second, $f$ is the fraction of electrons entered into the counter, $\tau$ is the dead time and $N_{\text {obs }}$ is the number of observed counting rate by the open counter. The photoemission yield was derived by dividing $N_{\text {in }}$ by the number of incident photons, which were counted using a photodiode (Model S1227-1010BQ, Hamamatsu Photonics) placed at the position of the sample.

\section{Results and Discussion}

At first, we examined changes in the photoemission yield spectra owing to the concentration of catechins using EGCg. Typical photoemission yield spectra of four different concentrations of EGCg are shown in Fig. 3. Although the photoemission yield measurement was repeated three times per sample, changes with time and the influence of damage by UV irradiation during the measurement were confirmed to be negligibly small. As shown in Fig. 3, the photoemission yield increased monotonically with an increase in the energy of incident photons. Monjushiro et al. reported that no photoemission was observed from pure water. $^{13}$ The photoemission spectrum of the pure water surface, which is shown in Fig. 3, exhibits the same trend. It can be seen that the photoemission yield increases with an increase in the concentration of EGCg. Prior to discussing the photoemission rates, the threshold energy of photoemission $E_{\mathrm{PET}}$ was determined.

The value of $E_{\mathrm{PET}}$, which corresponds to the first photoionization potential, was determined from the energy of an intersecting point between the background line and the extrapolated line of the linear portion of cube root plots of the photoemission yield. As shown in Fig. 4, it is clear that the value of $E_{\mathrm{PET}}$ can be determined from the plot because of its good linearity. As can be seen in Fig. 4, the value of $E_{\mathrm{PET}}$ is almost constant at $5.46 \pm 0.02 \mathrm{eV}$. According to this fact, the photoemission yield at a certain excitation energy was utilized for quantitative analysis. Figure 5 shows the dependence of the photoemission yield at $6.0 \mathrm{eV}$ upon the concentration of EGCg. Each point represents the average of three replicate measurements. 


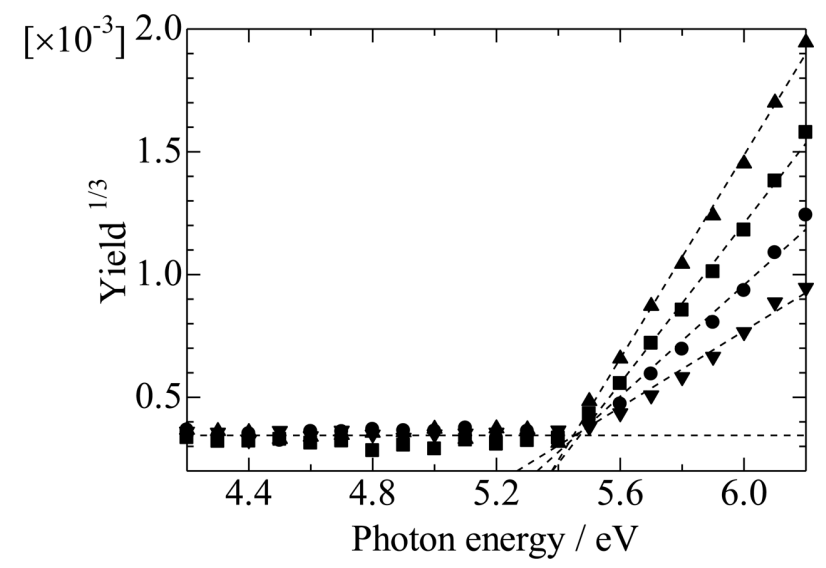

Fig. 4 Cube root plots of the photoemission yield of epigallocatechin gallate at the concentrations of $1000(\mathbf{\Lambda}), 500(\boldsymbol{\square}), 250(\mathbf{\bullet})$, and 100 ( $) \mathrm{mg} / \mathrm{L}$.

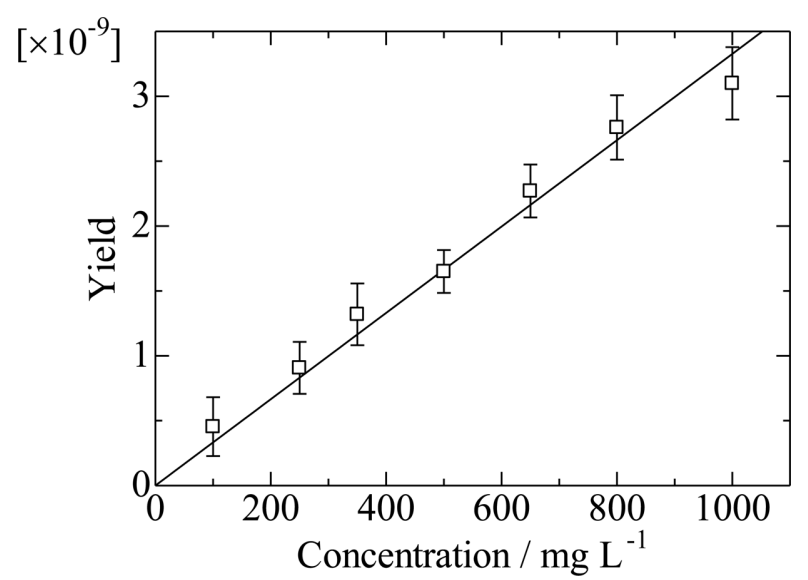

Fig. 5 Dependence of the photoemission yield at $6.0 \mathrm{eV}$ on the concentration of the epigallocatechin gallate.

The plot is almost a straight line, indicating that the photoemission yield is proportional to the concentration of EGCg. These facts indicate that catechins are evenly distributed and the state of catechins does not depend on the concentration.

Next, we measured EC, EGC, and ECg. The concentration was $500 \mathrm{mg} / \mathrm{L}$, which is based on the general amount in commercial bottled green tea. Because $500 \mathrm{mg} / \mathrm{L}$ indicates the total amount of catechins, the use of this value is merely an indication. Cube root plots of the photoemission yield for EC, EGC, ECg, and EGCg are shown in Fig. 6. The values of $E_{\mathrm{PET}}$ for EC, EGC, and ECg were estimated to be $5.72 \pm 0.02$, $5.68 \pm 0.01$, and $5.45 \pm 0.02 \mathrm{eV}$, respectively. As mentioned above, the value of $E_{\mathrm{PET}}$ for EGCg was $5.46 \pm 0.02 \mathrm{eV}$. It is clear that catechins with a galloyl moiety have a smaller value of $E_{\mathrm{PET}}$. According to several reports, ${ }^{14-18} \mathrm{ECg}$ and EGCg show higher biological activities than EC and EGC. With respect to the mechanism of their biological effects, activities as both anti-oxidants ${ }^{25,26}$ and pro-oxidants ${ }^{27,28}$ have been suggested. In either case, however, the oxidation of catechins seems to be an important reaction. From the viewpoint of electron transfer, oxidation is a chemical reaction involving the loss of electrons. While this study cannot provide a detailed discussion, the oxidation of catechin could be affected by the value of $E_{\mathrm{PET}}$.

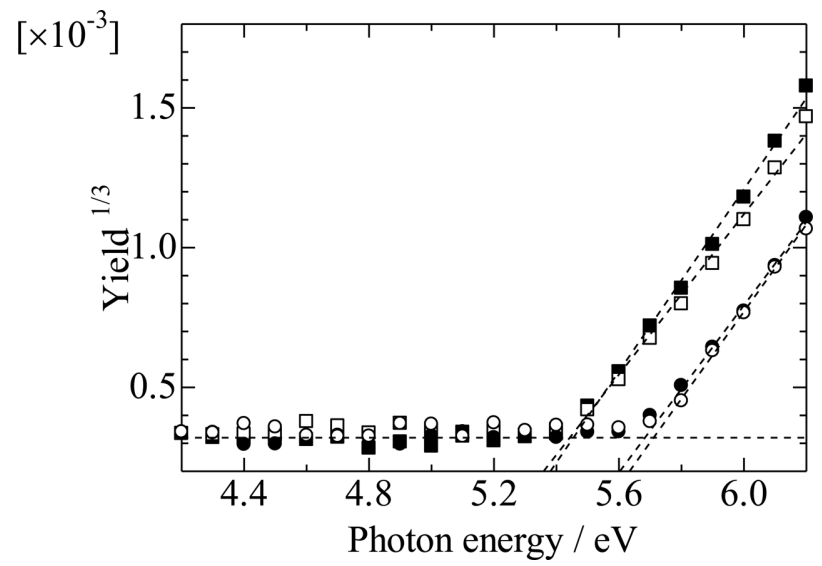

Fig. 6 Cube root plots of the photoemission yield of epicatechin $(\bigcirc)$, epigallocatechin $(\bullet)$, epicatechin gallate $(\square)$, epigallocatechin gallate (ם). Concentration of catechins was $500 \mathrm{mg} / \mathrm{L}$.

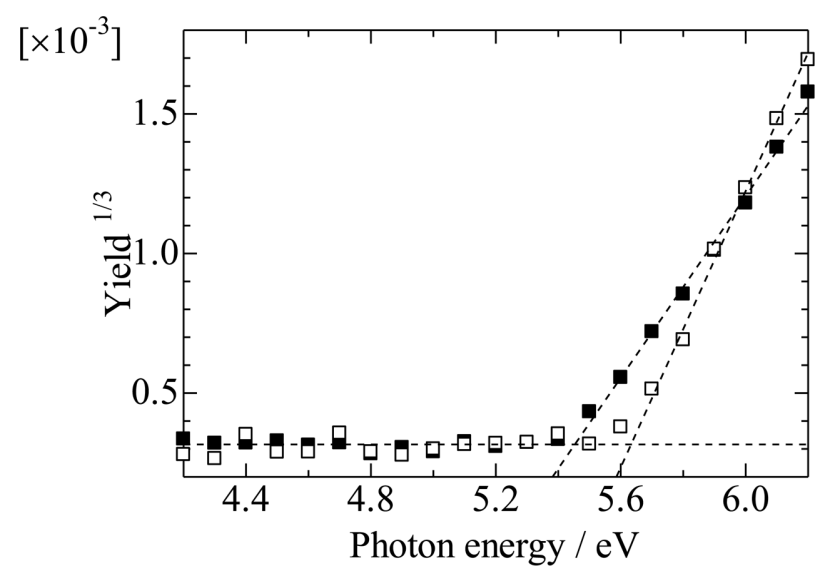

Fig. 7 Cube root plots of the photoemission yield of epigallocatechin gallate. Solid squares ( $\boldsymbol{\square})$ denote the results for the epigallocatechin gallate in water. Open squares $(\square)$ denote the results for the heated epigallocatechin gallate in water.

Moreover, the slope of the photoemission yield seems to be almost constant.

When pure $\mathrm{EGCg}$ was autoclaved at $120^{\circ} \mathrm{C}$ for $20 \mathrm{~min}$, the epimerization of EGCg to GCg was observed. ${ }^{31}$ Then, we confirmed a change in the photoemission yield spectra. After $20 \mathrm{~min}$ of heating, the powder was mixed with pure water. We prepared an aqueous solution of $500 \mathrm{mg} / \mathrm{L}$ and compared the photoemission yield spectra. As can be seen in Fig. 7, the differences in the $E_{\mathrm{PET}}$ and in the slope are remarkable. To elucidate these results, we also carried out the molecular orbital calculations. We constructed two cluster models for cis- and trans-type forms, i.e., EGCg and GCg. The Firefly QC package, ${ }^{32}$ which is partially based on the GAMESS (US) ${ }^{33}$ source code, was employed. The B3LYP hybrid functional ${ }^{34-37}$ with the $6-31 \mathrm{G}(\mathrm{d}, \mathrm{p})$ basis set was used for determining optimized geometries and their energies. The polarizable continuum model ${ }^{38}$ was adopted in the solvent (water). In this method, a solute is placed in a cavity formed by a union of spheres centered on each atom. The calculated occupied molecular orbitals for the ground state are shown in Fig. 8. When the energy of the HOMO decreases, the photoemission threshold should increase. Therefore, our present molecular 


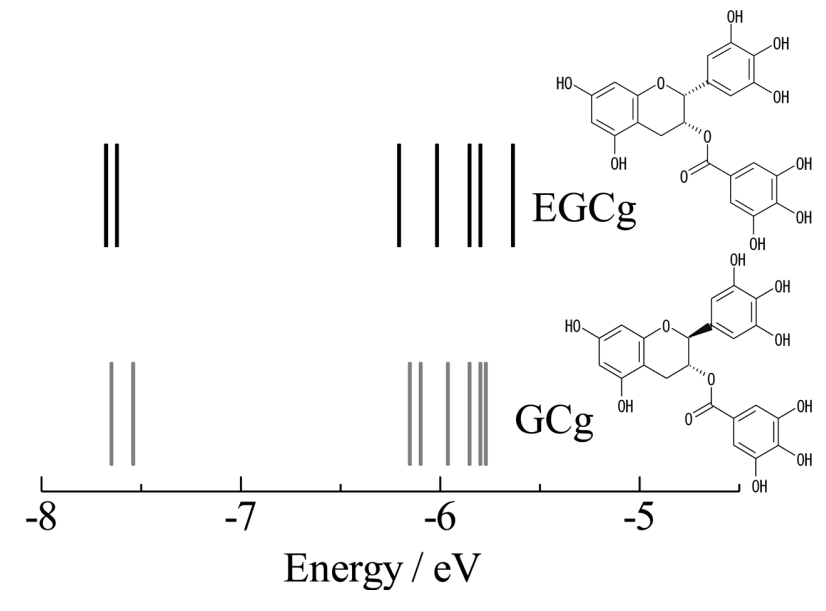

Fig. 8 Results of molecular orbital calculations for epigallocatechin gallate $(\mathrm{EGCg})$ and gallocatechin gallate $(\mathrm{GCg})$.

orbital calculations could explain the change in the threshold energy of photoemission caused by the epimerization of EGCg to $\mathrm{GCg}$. Furthermore, the molecular orbitals of $\mathrm{GCg}$ are denser around the HOMO than those of EGCg. This fact implies that the slope of the photoemission yield should slightly increase owing to epimerization. Consequently, the differences in the observed photoemission yield spectra are well explained by the present models.

\section{Conclusions}

In this study, PYSA was applied to the characterization of catechins in water. Photoemission measurements for characterizing the electronic properties of catechins in water have been successfully performed under the same conditions as our living environment. Although the photoemission rates vary with the concentration of epigallocatechin gallate, threshold energies of photoemission are almost the same. Contrarily, threshold energies of photoemission are classified into two groups: those for gallic acid esters of catechins and those for catechins without a galloyl moiety. This classification resembles the trend in their biological activities. Furthermore, change in the photoemission yield spectra, which corresponds to the epimerization of EGCg to $\mathrm{GCg}$, was observed. Molecular orbital calculations were also carried out to obtain changes in electronic structures, which support the results of PYSA. It is concluded that PYSA is a useful technique for characterizing the electronic properties of catechins in water. We hope that PYSA can provide useful information regarding the mechanisms of biological action.

\section{References}

1. H. Kirihata and M. Uda, Rev. Sci. Instrum., 1981, 52, 68.

2. M. Uda, Jpn. J. Appl. Phys., 1985, 24, 284.

3. T. Noguchi, S. Nagashima, and M. Uda, Nucl. Instrum. Meth. A, 1994, 342, 521.

4. S. Nagashima, T. Tsunekawa, N. Shiroguchi, H. Zenba, and M. Uda, Nucl. Instrum. Meth. A, 1996, 373, 148.

5. M. Uda, Y. Nakagawa, T. Yamamoto, M. Kawasaki, A. Nakamura, T. Saito, and K. Hirose, J. Electron Spectrosc. Relat. Phenom., 1998, 88-91, 767.
6. D. Yamashita, A. Ishizaki, and T. Yamamoto, Anal. Sci., 2014, 30, 575.

7. D. Yamashita, A. Ishizaki, and T. Yamamoto, Mater. Trans., 2015, 56, 1445.

8. H. Siegbahn and K. Siegbahn, J. Electron Spectrosc. Relat. Phenom., 1973, 2, 319.

9. M. Faubel, B. Steiner, and J. P. Toennies, J. Chem. Phys., 1997, 106, 9013.

10. H. Siegbahn, S. Svensson, and M. Lundholm, J. Electron Spectrosc. Relat. Phenom., 1981, 24, 205.

11. I. Watanabe, Anal. Sci., 1994, 10, 229.

12. K. Maya, I. Watanabe, and S. Ikeda, J. Electron Spectrosc. Relat. Phenom., 1986, 40, 307.

13. H. Monjushiro, K. Harada, and M. Haga, Langmuir, 2003, 19, 9226.

14. Y. Hara and M. Watanabe, Nippon Shokuhin Kogyo Gakkaishi, 1989, 36, 951.

15. S. Miura, J. Watanabe, T. Tomita, M. Sano, and I. Tomita, Biol. Pharm. Bull., 1994, 17, 1567.

16. T. Ishikawa, M. Suzukawa, T. Ito, H. Yoshida, M. Ayaori, M. Nishiwaki, A. Yonemura, Y. Hara, and H. Nakamura, Am. J. Clin. Nutr., 1997, 66, 261.

17. M. Tezuka, H. Suzuki, Y. Suzuki, Y. Hara, and S. Okada, Jpn. J. Toxicol. Environ. Health, 1997, 43, 311.

18. S. Okabe, M. Suganuma, M. Hayashi, E. Sueoka, A. Komori, and H. Fujiki, Jpn. J. Cancer Res., 1997, 88, 639.

19. M. Nakayama, K. Suzuki, M. Toda, S. Okubo, Y. Hara, and T. Shimamura, Antiviral Res., 1993, 21, 289.

20. A. Yamaguchi, M. Honda, H. Ikigai, Y. Hara, and T. Shimamura, Antiviral Res., 2002, 53, 19.

21. A. Hamza and C. Zhan, J. Phys. Chem. B, 2006, 110, 2910.

22. S. Wolfram, Y. Wang, and F. Thielecke, Mol. Nutr. Food Res., 2006, 50, 176.

23. B. N. Singh, S. Shankar, and R. K. Srivastava, Biochem. Pharmacol., 2011, 82, 1807.

24. S. F. Kostin, D. E. McDonald, and D. W. McFadden, J. Surg. Res., 2012, 177, 255.

25. A. Chen and L. Zhang, J. Biol. Chem., 2003, 278, 23381.

26. S. K. Katiyar, F. Afaq, A. Perez, and H. Mukhtar, Carcinogenesis, 2001, 22, 287.

27. G. X. Li, Y. K. Chen, Z. Hou, H. Xiao, H. Jin, G. Lu, M. J. Lee, B. Liu, F. Guan, Z. Yang, A. Yu, and C. S. Yang, Carcinogenesis, 2010, 31, 902.

28. G. Y. Yang, J. Liao, C. Li, J. Chung, E. J. Yurkow, C. T. Ho, and C. S. Yang, Carcinogenesis, 2000, 21, 2035.

29. R. Wang, W. Zhou, and X. Jiang, J. Agric. Food Chem., 2008, 56, 2694.

30. J. Z. Xu, L. K. Leung, Y. Huang, and Z. Y. Chen, J. Sci. Food Agric., 2003, 83, 1617.

31. Z. Y. Chen, Q. Y. Zhu, D. Tsang, and Y. Huang, J. Agric Food Chem., 2001, 49, 477.

32. A. A. Granovsky, Firefly, www http://classic.chem.msu.su/ gran/firefly/index.html.

33. M. W. Schmidt, K. K. Baldridge, J. A. Boatz, S. T. Elbert, M. S. Gordon, J. H. Jensen, S. Koseki, N. Matsunaga, K. A. Nguyen, S. Su, T. L. Windus, M. Dupuis, and J. A. Montgomery, J. Comput. Chem., 1993, 14, 1347.

34. A. D. Becke, J. Chem. Phys., 1993, 98, 5648.

35. C. Lee, W. Yang, and R. G. Parr, Phys. Rev. B, 1988, 37, 785.

36. S. H. Vosko, L. Wilk, and M. Nusair, Can. J. Phys., 1980, $58,1200$.

37. P. J. Stephens, F. J. Devlin, C. F. Chabalowski, and M. J. Frisch, J. Phys. Chem., 1994, 98, 11623.

38. S. Miertus, E. Scrocco, and J. Tomasi, Chem. Phys., 1981, $55,117$. 